\title{
Reducing Long-term Opioid Use in the Veterans Health Administration
}

\author{
Taeko Minegishi, $\mathrm{MS}^{1,2,3}$ and Austin Frakt, $P h D^{1,2,4}$

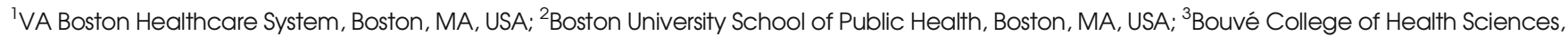 \\ Northeastern University, Boston, MA, USA; ${ }^{4}$ Harvard T.H. Chan School of Public Health, Boston, MA, USA.
}

J Gen Intern Med 33(6):781-2

DOI: $10.1007 / \mathrm{s} 11606-018-4352-7$

(c) Society of General Internal Medicine (outside the USA) 2018

I n 2015, 25\% of US adults had at least one opioid prescription. In the same year, over 33,000 people died of opioids and approximately $40 \%$ were attributed to opioid prescriptions, ${ }^{1}$ contributing to what the Centers for Disease Control and Prevention (CDC) termed an "epidemic." Long-term opioid use is particularly problematic, associated with much higher rates of misuse and overdose than short-term use. ${ }^{2}$ As we reconsider the appropriate role of opioids in medical care, experts have called for an effort to minimize the initiation of long-term use and to taper long-term patients off opioids, when warranted.

The opioid crisis is also evident at the Veterans Health Administration (VHA). Fifty percent of chronic non-cancer pain patients at the VHA received at least one opioid prescription each year between 2009 and $2011 .^{3}$ Compared to the general US population, VHA patients had nearly twice the rate of fatal accidental poisoning and opioid use disorder is seven times higher among VHA patients compared to private health plans. ${ }^{3}$ Greater risk of opioid use within the VHA than in the broader health system can be explained by the fact that VHA patients are more likely to suffer from chronic pain $(50 \%$ do so) and more of its patients have psychiatric comorbidities. Among US veterans of Iraq and Afghanistan, mental health diagnoses, especially post-traumatic stress disorder, were associated with an increased risk of receiving opioids for pain, high-risk opioid use, and adverse clinical outcomes. ${ }^{4}$

There is some encouraging news that suggests we may be turning the tide on overuse of prescription opioids. Both nationally and in the VHA, since 2012, opioid prescribing has decreased. In 2015, it was down $13 \%$ nationally, and in 2016, it was down 25\% in the VHA, both relative to 2012 levels. ${ }^{5}$ Nevertheless, the number of opioid prescriptions remains high by historical standards. Three times more opioids were prescribed in 2015 than in 1999. Nationally, the recent reduction in prescribing is attributed to lower rates of short-term use. Long-term use remains high and a significant concern.

Published online February 15, 2018
This is where the VHA's experience offers considerable hope. Based on a cohort of patients with any prescriptions (about five million patients per year), the study by Hadlandsmyth et al. ${ }^{6}$ shows that the VHA's reduction in opioid prescribing has been led by lower rates of long-term opioid use. The investigators classified the duration of opioid use into four categories: no use, short-term use (single opioid prescription or two prescriptions separated by $>90$ days), long-term use ( $>90$ days), and intermediate-term use (anything else). Between 2010 and 2016, reduced long-term use among VHA patients accounted for $83 \%$ of the downturn in opioid prescribing.

Consistent with national trends, Hadlandsmyth et al. ${ }^{6}$ found that the prevalence of overall opioid prescribing declined between 2010 and 2016, from 21 to 16\%. Short-term prescribing fell only $1.6 \%$, but long- and intermediate-term prescribing fell 25 and $32 \%$, respectively. More than $90 \%$ of the reduction in the VHA long-term opioid use can be attributed to the reduction in the number of new long-term users, not discontinuation of existing long-term users.

To what can we attribute the VHA's apparent greater success in reduced long-term opioid use, relative to the national trend? First, VHA clinicians may be better equipped with resources that discourage them from initiating long-term opioid therapy. The joint Department of Veterans Affairs/Department of Defense Clinical Practice Guideline for Management of Opioid Therapy for Chronic Pain recommends against initiating longterm opioid therapy. And, in 2009, VHA leadership issued directives that expanded access to complementary treatments and multimodal therapy for chronic pain.

Second, leveraging the VHA's data capabilities, the VHA Opioid Safety Initiative (OSI) included an effort to promote safer opioid-related prescribing. The Initiative tracks urine drug screens, incorporates state prescription drug monitoring databases, and aims to reduce high-dose and concurrent benzodiazepine prescribing within the VHA.

Third, the VHA provides academic detailing - a service for clinicians by clinical pharmacy specialists that provides individualized, face-to-face outreach to encourage evidence-based decision-making to improve veterans' health. ${ }^{7}$ For example, VHA academic detailing may promote the use of an opioid taper decision tool and of follow-up and support during opioid tapering. 
Going forward, the VHA is initiating other programs to help reduce unnecessary opioid prescribing, and to promote mitigation strategies for patients that require opioids. For example, the Stratification Tool for Opioid Risk Mitigation (STORM) is a dashboard developed to help identify to VHA clinicians opioid-prescribed patients at high risk of adverse events. ${ }^{8}$ In addition to patients' medical history and prescription information, the dashboard recommends risk mitigation strategies and non-pharmacological pain treatment approaches. Starting in the spring of 2018, the VHA is launching a randomized controlled evaluation assessing the clinical impact of the STORM dashboard. ${ }^{9}$

Efforts like these are needed nationally. Previous research has shown that among high-dose long-term opioid users, $80 \%$ remained high-dose opioid patients 1 year later even though half of the users expressed a desire to reduce or terminate opioid medications. ${ }^{10}$ Therefore, it is crucial that we help patients appropriately reduce opioid use earlier through safe tapering. Unfortunately, the evidence based on safe tapering methods is thin. According to recent systematic reviews, many studies examining interventions for dose reduction or discontinuation of long-term opioid use are of poor quality with uncontrolled designs. ${ }^{2}$

Hadlandsmyth et al. ${ }^{6}$ and other studies have shown promising developments in fighting the opioid crisis. Nonetheless, there is more work to do. Their study only included VHAprescribed opioids, so opioids prescribed outside of the VHA were not accounted for. To more thoroughly understand the trends in long-term opioid discontinuation and tapering, linked VHA and non-VHA medical data are necessary. Medicare and Medicaid research claims data are usually at least a year old and do not allow for prospective studies. Recent progress in linking VHA and Department of Defense data allows for expanding the population to active and retired military personnel. By integrating patients' pain levels and medication history during active military services, VHA clinicians may be better prepared to identify high-risk patients for long-term opioid use. Furthermore, it is unclear as to what degree the VHA's experience in reducing opioid prescribing in general, and long-term opioid use in particular, can be attributed to the programs it has implemented. Rigorous evaluations are required to assess their specific impacts and to draw conclusions about whether and how to diffuse them beyond the VHA.

Most importantly, the effectiveness and risks of opioid tapering need close examination. We do not know if patients tapered off long-term opioid use are at higher risk of adverse events or experience uncontrolled pain. As we encourage both VHA and non-VHA clinicians to taper long-term opioid users off their pain medications, we must be sure they are doing so safely and to the benefit of patients. Reductions in long-term use that also lead to increases in serious adverse events or debilitating levels of pain would be a hollow victory.

Corresponding Author: Taeko Minegishi, MS; Bouvé College of Health SciencesNortheastern University, Boston, MA, USA (e-mail: taeko.minegishi@va.gov).

Funding This work is supported by Department of Veterans Affairs, Veterans Health Administration, Office of Research and Development (GUERI PEC 16-001). The contents do not represent the views of the U.S. Department of Veterans Affairs, the United States Government, Northeastern University, Boston University, or Harvard University.

\section{Compliance with ethical standards:}

Conflict of interest: None.

\section{REFERENCES}

1. Center for Disease Control and Prevention. Annual Surveillance Report of Drug-Related Risks and Outcomes - United States, 2017. Centers for Disease Control and Prevention, U.S. Department of Health and Human Services; 2017.

2. Frank JW, Lovejoy TI, Becker WC, et al. Patient outcomes in dose reduction or discontinuation of long-term opioid therapy: a systematic review. Ann Intern Med. 2017;167(3):181-191.

3. Edlund MJ, Austen MA, Sullivan MD, et al. Patterns of opioid use for chronic noncancer pain in the Veterans Health Administration from 2009 to 2011: Pain. 2014; 155(11):2337-2343.

4. Seal KH, Shi Y, Cohen G, et al. Association of mental health disorders with prescription opioids and high-risk opioid use in US veterans of Iraq and Afghanistan. JAMA. 2012;307(9):940-947.

5. Gellad WF, Good CB, Shulkin DJ. Addressing the opioid epidemic in the United States: lessons from the Department of Veterans Affairs. JAMA Intern Med. 2017;177(5):611-612.

6. Hadlandsmyth K, Mosher H, Vander Weg MW, Lund BC. Decline in prescription opioids attributable to decreases in long-term use: a retrospective study in Veterans Health Administration 2010-2016. J Gen Intern Med. https://doi.org/10.1007/s11606-017-4283-8.

7. Gallagher RM. Advancing the pain agenda in the Veteran population. Anesthesiol Clin. 2016;34(2):357-378.

8. Minegishi T, Frakt AB, Garrido $\mathbf{M M}$, et al. Randomized program evaluation of the VHA Stratification Tool for Opioid Risk Mitigation (STORM): a research and clinical operations partnership to examine effectiveness. Subst Abuse. 2018 (in press).

9. Minegishi T, Garrido MM, Pizer SD, Frakt AB. The Effectiveness of Policy and Risk Targeting for Opioid-Related Risk Mitigation: A Randomized Program Evaluation. BMJ Open. 2018 (in press).

10. Thielke SM, Turner JA, Shortreed SM, et al. Do patient-perceived pros and cons of opioids predict sustained higher-dose use? Clin J Pain. March 2013:1. 